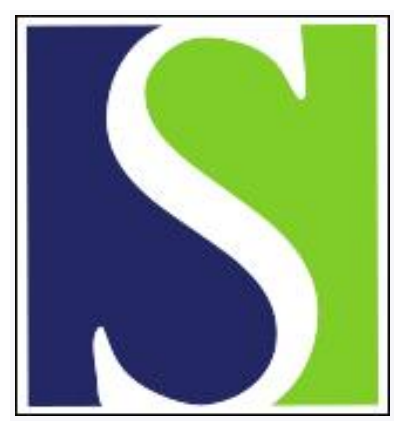

Scand J Work Environ Health 2003;29(6):452-460

https://doi.org/10.5271/sjweh.753

Issue date: Dec 2003

Physiological responses to four hours of low-level repetitive work

by Garde AH, Hansen ÅM, Jensen BR

Affiliation: National Institute of Occupational Health, Lersø Parkallé, DK-2100 Copenhagen, Denmark. ahg@ami.dk

Refers to the following text of the Journal: 2002;28(4):215-221

Key terms: electromyography; epinephrine; hydrocortisone; low-level physical demand; low-level repetitive work; mental demand; norepinephrine; physiological response

This article in PubMed: www.ncbi.nlm.nih.gov/pubmed/14712853

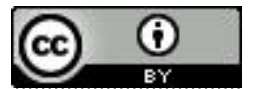




\title{
Physiological responses to four hours of low-level repetitive work
}

\author{
by $A$ Helene Garde, PhD, ${ }^{1}$ Åse M Hansen, PhD, ${ }^{1}$ Bente $R$ Jensen, $P h D^{2}$
}

\begin{abstract}
Garde AH, Hansen ÅM, Jensen BR. Physiological responses to four hours of low-level repetitive work. Scand J Work Environ Health 2003;29(6):452-460.

Objectives The study investigated physiological responses to 4 hours of standardized low-level repetitive work. It was hypothesized that accumulative effects not observed after 1 hour could be found after 4 hours of repetitive work.

Methods Ten healthy women performed intermittent ( 5 seconds +5 seconds) handgrip contractions at $10 \%$ of the maximal voluntary contraction combined with mental demands for concentration and attention. Muscle activity in the working forearm muscles, cardiovascular responses, and concentrations of biomarkers in biological fluids were recorded along with exerted force, performance, and ratings of perceived physical exertion (RPE), and perceived mental exertion.

Results The urinary epinephrine, norepinephrine, and cortisol concentrations were higher during the repetitive task than on a reference day, but only the norepinephrine concentrations increased progressively during the 4 hours. In accordance, the RPE recorded for the hand, forearm, and shoulder regions increased progressively. For the remaining physiological measures, no accumulative changes were found. Forearm muscle activity was higher during a mental reference task with lower exerted force than during the repetitive task. The variation in exerted force was higher during the repetitive task than during a force reference task without mental demands. Conclusions The urinary biomarkers were increased during the repetitive task. However, only norepinephrine increased progressively during the 4 hours. Forearm muscle activity during a mental reference task with low exerted force indicated attention-related muscle activity. Finally, it was indicated that repetitive work including high demands for attention is performed at the expense of the precision of the exerted force.
\end{abstract}

Key terms electromyography, epinephrine, hydrocortisone, low-level physical demands, mental demands, norepinephrine.

Repetitive work in occupational settings often implies a combination of mental and physical demands for up to 8 hours a day. The physiological responses to dynamic whole-body physical demands such as running or cycling are well established for concentrations of epinephrine, norepinephrine, and cortisol (1), and they are known to some degree for adrenocorticotrophic hormone (ACTH), dehydroepiandrosterone sulfate (DHEA$\mathrm{S})$, prolactin, and testosterone $(2,3)$, whereas the physiological responses to low-level physical demands such as dynamic handgrip work are less clear $(4,5)$.

In field studies, urinary concentrations of epinephrine and norepinephrine have been shown to increase in response to repetitive work $(6,7)$. This finding has been confirmed in several laboratory studies (8-10), although other results are contradictory (11-13). Differences in the physical and mental demands or the duration of the exposure may potentially explain the discrepancies.
Most studies were carried out for less than 2 hours, whereas only a few studies measuring urinary catecholamines in response to standardized repetitive work have been conducted for more than 2 hours $(9,10)$. Thus the potential accumulative effects on physiological measures and perceived physical and mental exertion during prolonged low-level exposure have not been fully elucidated.

Several studies have shown increased muscle activity in response to mental demands, as reviewed by Wærsted (14). It has been debated in the literature whether some muscles are more sensitive to mental demands during work than others. However, a recent study indicated that the effect of mental demands on muscle activity is more general and involves all active muscles; this finding can be explained by increased central command (15). However, the knowledge regarding potential accumulative effects due to exposure to mental demands for several hours is limited.

1 Department of Physiology, National Institute of Occupational Health, Copenhagen, Denmark.

2 Department of Human Physiology, Institute of Exercise and Sport Sciences, University of Copenhagen, Denmark.

Reprint requests to: Dr A Helene Garde, National Institute of Occupational Health, Lers $\emptyset$ Parkallé 105, DK-2100 Copenhagen, Denmark. [E-mail: ahg@ami.dk] 


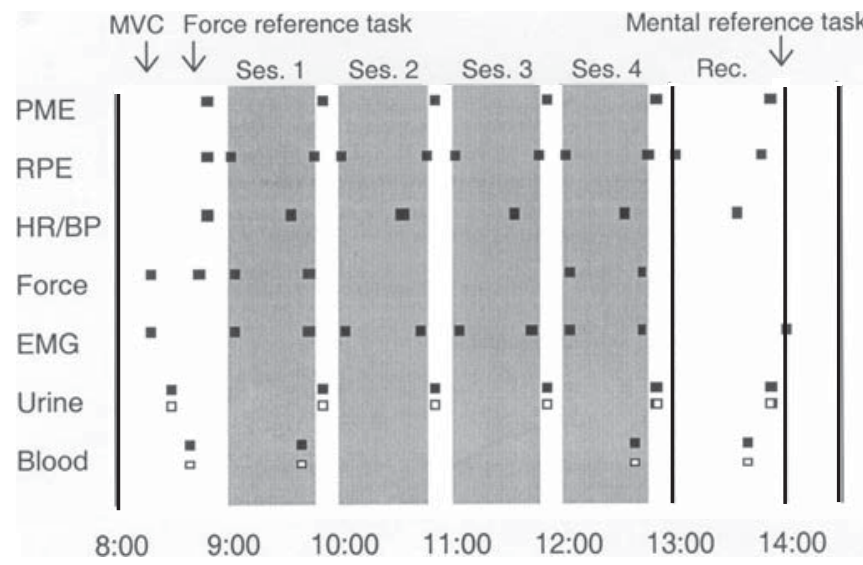

Figure 1. Overview of the data collection. The gray areas indicate periods with a repetitive task. Closed squares indicate that data were collected on the experimental day, and open squares represent data collected on the reference day. (MVC = maximal voluntary contraction, $\mathrm{PME}=$ perceived mental exertion, $\mathrm{RPE}=$ rating of perceived physical exertion, $\mathrm{HR}=$ heart rate, $\mathrm{BP}=$ blood pressure, $\mathrm{EMG}=$ electromyography, Ses. = session, $\mathrm{Rec} .=$ recovery)

The purpose of our study was to investigate physiological responses to 4 hours of standardized low-level repetitive work including physical and mental components. It was hypothesized that accumulative effects not observed after 1 hour would be found after 4 hours of low-level repetitive work.

\section{Subjects and methods}

\section{Study group}

Ten healthy female students with no pain or discomfort in the upper extremities, neck, or low back for at least the past 5 months participated in the study [age: mean 24 (range 20-31) years, height: mean 168 (range 157-175) cm, weight: mean 61 (range 51-68) kg]. All the participants gave their written informed consent to participate. Exclusion criteria were pregnancy, lactation, or use of medication, including contraceptive pills or other forms of a hormone preparation on a regular basis. The subjects were asked to refrain from heavy exercise the days before coming to the laboratory, and they were encouraged to eat breakfast without drinking coffee before arriving at the laboratory.

\section{Procedure}

On the experimental day a repetitive task was performed between 0900 and 1430. The repetitive task was performed during four 50-minute sessions, separated by 10 minute pauses and followed by 1 hour of recovery. The task was to perform intermittent handgrip contractions (right hand) while solving attention-demanding computer problems using voice responses (DragonDictate, Starter Edition, British English, Dragon Systems UK Ltd., Milton, United Kingdom). An overview of the procedure and data collection is given in figure 1 .

The intermittent handgrip contractions consisted of 5second contractions at $10 \%$ of the maximal voluntary

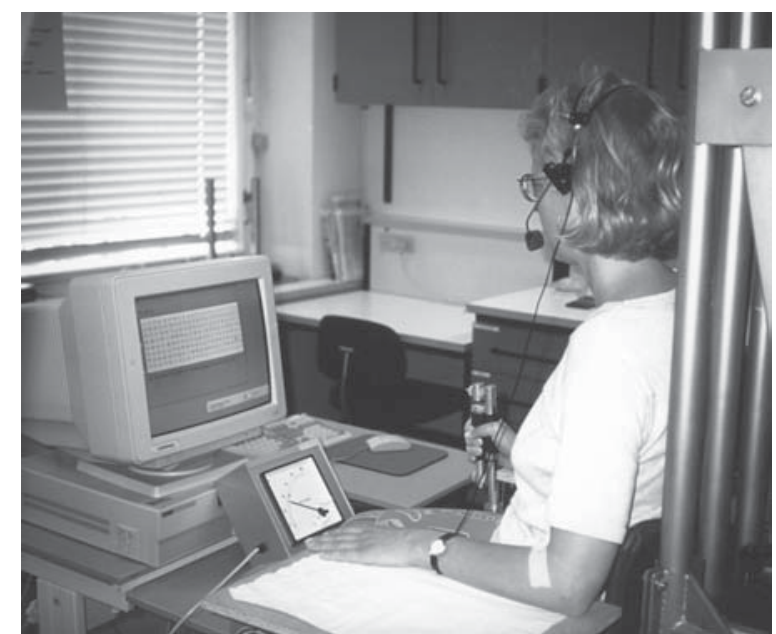

Figure 2. The repetitive task.

contraction (MVC) alternated with 5 seconds of rest. A brief sound was given every 5 seconds to indicate shifts between contractions and rest. The handgrip transducer was fixed to the table in a vertical position (figure 2), and the subjects performed the handgrip task in a sitting position. The elbow was flexed at an angle of 90 degrees with the forearm horizontal and the thumb up. The forearm was fully supported by a table, the height of which was adjusted for each subject. The participants were provided with visual feedback of the exerted force and were asked to perform the handgrip task as precisely as possible.

The attention-demanding computer task consisted of three types of tasks ("dots", "brick-in-frame", and "number finding") performed in a fixed order $(16,17)$. In "dots", groups of 3 to 5 dots were presented to the participant, who answered "yes" or "no" to the question: "Are there exactly 4 dots in this group?" Performance was recorded as the number of errors relative to the total number of answers. In "brick-in-frame" a $5 \times 7$ square grid with 1 brick and 1 frame in 2 separate squares was presented, together with a statement (eg, "4 left and 1 up") indicating the number of squares the brick must be moved to the left or right and up or down 
to make it cover the frame. The participants were to report whether the statement was correct by answering "yes" or "no". Performance was recorded as the number of errors relative to the total number of answers and the time spent on each item. In "number finding" the participants were presented with an $8 \times 8$-square grid with the numbers between 1 and 64 in random order. Each square contained two numbers, one (cue) in a large font and the other (associated number) in a small font. The participants identified the cues in numerical order and reported the associated number by use of the digits 0 9. No performance measures were recorded. Additional details of the tests have been reported earlier $(16,17)$. Each item was visible until the participants responded, and immediately thereafter a new item was presented. The participants were encouraged to answer as correctly and as quickly as possible.

As reference, urine and blood were collected on a separate reference day, on which the participants were seated relaxed at a desk in order to control for diurnal variation. The order of the experimental day and reference day was randomized. Food intake (light sandwich and fruit) was standardized as during the experimental day. Furthermore, the participants performed a force reference task consisting of 2 minutes of the intermittent handgrip contractions without the mentally demanding task. Thus the force reference task included the physical, but not the mental, component of the repetitive task. A mental reference task consisted of 5 minutes of the "dots" task, but the keyboard was used rather than voice response (figure 3). The mental reference task was performed with the participant sitting at a computer table with the height of the workstation adjusted to the participant, but with no support of the forearm. Thus the mental reference task included the mental component of the repetitive task, whereas the externally required force (ie, the force applied to the keyboard) was

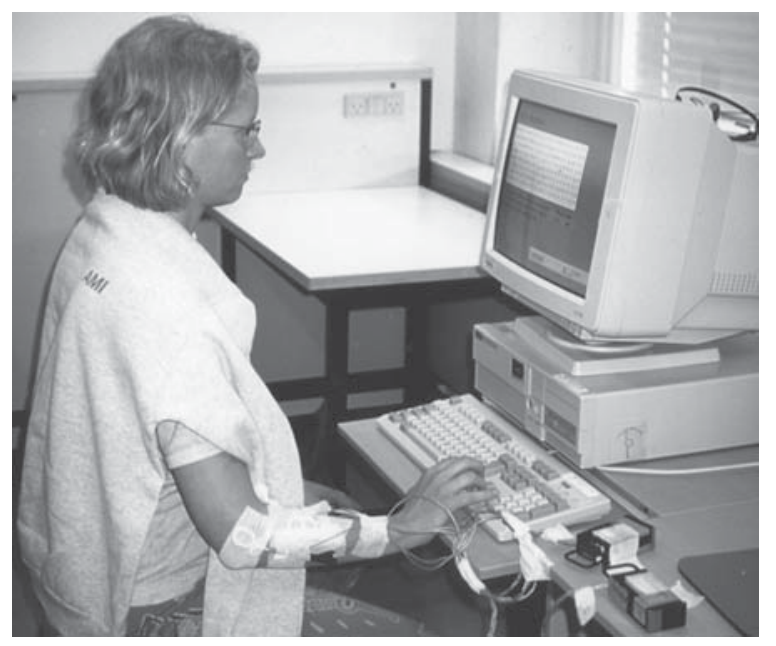

Figure 3. The mental reference task. smaller (18) than during the repetitive task. The mentally and physically demanding tasks were practiced on another separate day before the experimental day.

\section{Performance of the mental tasks}

Performance on the "dots" and "brick-in-frame" tasks was recorded once in each session.

\section{Perceived physical and mental exertion}

The rating of perceived physical exertion (RPE) was recorded for the hand, forearm, and shoulder regions according to a 10-grade (0 to 9) Borg scale (19) before the repetitive task, in the beginning of the task, and at the end of each 50-minute session (during and between contractions), and after 1 hour of recovery.

Perceived mental workload during the past hour was recorded using 17 visual analogue scales (0 to10) before the repetitive task, during the 10-minute pauses between the 50-minute sessions, and after 1 hour of recovery. With the use of two separate factor analyses, five factors with eigenvalues greater than 1 were found. Two factors $(60 \%$ of the total variance) described ratings of mood and three factors (74\% of the total variance) described ratings of the task. Higher scores indicated a higher degree of positive feelings (doing my best, being concentrated, being satisfied, being in control), negative feelings (feeling sad, feeling stressed, feeling irritated), boring task [interesting task (inverted), boring task, stupid task], demanding task (demanding task, difficult task, involving task), and funny task (funny task, childish task).

\section{Heart rate and blood pressure}

Heart rate, as well as systolic and diastolic blood pressure, were recorded every 60 seconds (2300 Finapress ${ }^{\mathrm{TM}}$ blood pressure monitor, BOC Ohmeda, Madison, WI, USA) on the nonworking hand of nine participants. The forearm was resting on a height-adjusted table with the elbow flexed at an angle of 90 degrees. A 5-minute mean was estimated for each participant before the repetitive task, 35 minutes into each 50-minute session, and after 20 minutes of recovery.

\section{Exerted force}

Prior to the beginning of the repetitive task, MVC during handgrip was measured as the highest 1-second value obtained in 3 to 5 attempts. The MVC was used for calculating the target force (10\% MVC). The actual exerted force was recorded on a tape recorder (Brüel and Kjær 7005, Nærum, Denmark). Periods of 100 seconds were sampled $(1024 \mathrm{~Hz})$ and analyzed off-line. The start 
of a 100-second recording period was synchronized in relation to the sound indicating a shift between contraction and rest, and the 100-second period was automatically divided into 5 -second periods. The middle 2 -second period within each 5-second period was analyzed. The exerted force was recorded during the force reference task and in the beginning and end of sessions 1 and 4 during the repetitive task. Performance was evaluated as (i) the mean exerted force during contractions, (ii) the variation of the exerted force during contractions within one 100-second recording period, and (iii) the number of mixed periods (in which the participant mixed contraction and rest within one 2 -second period).

\section{Electromyography}

Electromyography (EMG) was recorded from the extensor carpi radialis (ECR) and flexor carpi radialis (FCR) muscles by use of bipolar $\mathrm{Ag}-\mathrm{AgCl}$ surface electrodes (N-10-A, Medicotest, Ølstykke, Denmark) with a distance of 20 millimeters between the recording areas. Commercially available telemetric equipment (Medinik IC-600-C, Medinik AB, Örbyhus, Sweden) was used to record the EMG before the repetitive task, in the beginning and end of all the sessions of the repetitive task, and during the mental reference task. Data were stored on a tape-recorder (Brüel and Kjær 7005). During the recording, the quality of the signals was checked on an oscilloscope (Leader LBO-308S, Leader, Hazlet, NJ, USA). The raw EMG signal was filtered $(2-450 \mathrm{~Hz})$ prior to the sampling $(1024 \mathrm{~Hz})$, and 2-second periods were analyzed off-line after subdivision into 5 -second periods, as described for exerted force. The root mean square (RMS) amplitude of the recorded EMG was estimated during and between contractions. The RMS amplitude was normalized in relation to the maximal EMG $\left(\% \mathrm{EMG}_{\max }\right)$, determined simultaneously with the MVC. The mean power frequency was calculated as described by Kwatny et al (20) during the contractions. Due to incomplete data collection, results are presented for six participants.

\section{Urine samples}

Urine samples were collected for the catecholamine determinations in $10-\mathrm{ml}$ polystyrene tubes coated with $200 \mu \mathrm{g}$ of citric acid, and samples for the measurement of cortisol were collected in plain 10-ml tubes. Urine samples were collected from nine participants before the repetitive task, after each 50-minute session, and after the 1-hour recovery on the experimental day and at the same times of the day on the reference day. The samples were stored at $-20^{\circ} \mathrm{C}$ and analyzed within 2 months. In order to normalize results in relation to urine volume, we measured the concentrations of creatinine in the urine.
Simultaneous clean-up and high-performance liquid chromatography with on-line derivatization and fluorescence detection was used for measuring the urinary catecholamines (21). The concentrations of urinary cortisol were determined by radioimmunoassay (RIA) (coata-count kit, Diagnostic Products Corporation, Los Angeles, CA, USA). A 1470 Wizard gamma counter (Wallac, Turku, Finland) was used for measuring radioactivity. Creatinine was measured with a COBAS Mira autoanalyzer (Roche Diagnostic Systems, Basel, Switzerland) on the basis of Jaffe's reaction (22). The analytical variations (CVa) were $10 \%, 13 \%$, and $3 \%$ for catecholamines, cortisol, and creatinine, respectively. The analytical methods had been evaluated, and performance was continuously monitored by use of commercial matrix reference materials where possible, as well as by participation in external quality assessment schemes (23, 24).

\section{Blood samples}

When possible (7 participants), venous blood was collected with the use of a venflon catheter $(18 \mathrm{G} / 32 \mathrm{~mm})$ inserted into the antecubital vein of the nonexercising (left) arm. Between the samplings, the catheter was blocked with an obturator (18 G/32 mm). Blood (20 ml) was drawn using a sterile syringe and immediately transferred to a 10-ml plain Vacutainer tube (Becton, Dickinson \& Co, NJ, USA) for the preparation of serum and to a $10-\mathrm{ml}$ Vacutainer tube with EDTA (ethylene diamine tetraacetic acid) (Becton, Dickinson \& Co) for the preparation of plasma. Otherwise (3 participants), blood samples were drawn by use of the Vacutainer system (Becton, Dickinson \& Co). Venous blood was collected on the following four occasions: before the repetitive task, 40 minutes into the first and the fourth 50minute sessions, and after 40 minutes of recovery for 10 participants on the experimental day and at the same times of the day on the reference day.

Plasma was analyzed for ACTH $(\mathrm{CVa}=6 \%$ at 7.7 pmol/l) by immunoradiometric assay (DYNO test, Brahms Diagnostica GMBH, Berlin, Germany). Serum was analyzed for DHEA-S (CVa=10\% at $3.4 \mu \mathrm{mol} / \mathrm{l})$ by an in-house enzyme-linked immunosorbentassay (25). The RIA used for determining free testosterone ( $\mathrm{CVa}<10 \%$ in the whole range of the method) and prolactin $(\mathrm{CVa}=9 \%$ at $182 \mathrm{mIU} / \mathrm{l})$ in serum were coat-acount kits purchased from the Diagnostic Products Corporation. A 1470 Wizard gamma counter from Wallac (Turku, Finland) was used for measuring radioactivity. UNIMATE 3 (art. 073696 1) was used for the immunoturbidimetric analysis of iummoglobulin A ( CVa $<3.5 \%$ in the whole range of the method) in serum, which was carried out by use of a COBAS Mira Plus (Roche Diagnostic Systems). Performance was continuously 
monitored with the use of matrix reference materials and participation in external quality assessment schemes (26).

\section{Statistical methods}

The statistical analyses were carried out by use of the SAS ${ }^{\circledR}$ System ${ }^{\mathrm{TM}}$, version 8.02 (SAS Institute, Cary, NC, USA). Performance, perceived physical and mental exertion, heart rate, systolic and diastolic blood pressure, exerted force, EMG, and the concentrations of biomarkers were analyzed as dependent variables in models of repeated-measures analyses of variance with participant as a random factor and an autoregressive covariance structure by the use of the mixed procedure. The main hypothesis, that effects not observed after 1 hour could be detected after 4 hours, was tested using only data collected during the 4 hours of work on the experimental day. The continuous variable time of day was included as the independent factor in all the models. The model for RPE also included the variable contraction (during or between contractions) and beginning-end (beginning or end of session). In order to control for diurnal variation in the concentrations of the biomarkers, models including the variables day (experimental or reference day), time of day (only data collected during workhours) and the interaction term dayxtime of day were also tested. The same models were used to test whether 4 hours of the repetitive task was associated with increased concentrations of biomarkers in urine and blood when compared with the concentrations on the reference day. If there was no statistically significant effect of the interaction term, it was excluded in order to test for main effects. Due to nonnormal (skewed) distributions and heteroscedastic variances for the variables, "number of errors" and "biomarker concentration", these were analyzed on logarithmic scales. A paired t-test was used for comparing different tasks and specific times (morning and after restitution) on the experimental and the reference days. Levene's test was used for testing for variance homogeneity. Residual plots were used to identify potential outliers, deviations from normality, and skewed distributions. The results are presented as means and standard deviations unless otherwise noted.

\section{Results}

\section{Performance}

The observed number of errors for the "brick-in-frame" task $\{5.0 \%$ [95\% confidence interval $(95 \% \mathrm{CI}) 1.8-$ $14.2 \%]\}$ did not differ $(\mathrm{P}=0.85)$ between the sessions of the repetitive task, whereas the time spent at each problem decreased from 4.2 (SD 0.7) seconds during the first session to 3.8 (SD 0.9) seconds during session 4 $(\mathrm{P}<0.001)$. For the "dots" task the observed number of errors [2.3\% (95\% CI 0.7-6.9\%)] did not differ between the sessions of the repetitive task $(\mathrm{P}=0.19)$.

\section{Perceived physical and mental exertion}

The RPE for the hand, forearm, and shoulder regions increased progressively during the 4 sessions of the repetitive task (table 1). Furthermore, on the average, the RPE was 0.8 (SD 0.3), 1.3 (SD 0.3), and 1.4 (SD 0.3) points higher during contraction for the shoulder, arm, and hand regions, respectively, compared with the values between contractions (table 1). The RPE was higher for all the regions during the repetitive task when compared with the values after 1 hour of recovery.

The task was rated as progressively less demanding [-0.41 (SD 0.18) points/hour, $\mathrm{P}=0.028]$ and more boring [+0.45 (SD 0.26) points/hour, $\mathrm{P}=0.098$ ]. No progressive changes were observed during the repetitive task for the other ratings of mental exertion (table 1). The ratings of finding the task demanding were higher $(\mathrm{P}<0.001)$ during the repetitive task than before the beginning of the task. The ratings for finding the task boring $(\mathrm{P}=0.017)$ were higher during the performance of the repetitive task than after the 1 hour of recovery (table 1). The ratings of negative feelings were higher during the task than before $(\mathrm{P}=0.008)$ and after $(\mathrm{P}<0.001)$ the task. There were no differences in the ratings of positive feelings and finding the task funny.

\section{Heart rate and blood pressure}

There were no progressive changes in heart rate or systolic and diastolic blood pressure during the repetitive task. There was an increase $(\mathrm{P}=0.019)$ in diastolic blood pressure during the repetitive task [90 (SD 12) $\mathrm{mmHg}$ ] when compared with the corresponding values before and after the task [86 (SD 14) $\mathrm{mmHg}$ ], while there were no differences for heart rate [69 (SD 6) beats/min] or systolic blood pressure [146 (SD 17) $\mathrm{mmHg}$ ].

\section{Exerted force}

A contraction level of $10 \%$ MVC corresponded to 27.5 (SD 4.7) N. The mean exerted forces did not change during the repetitive task $(\mathrm{P}=0.56)$. Furthermore, no statistical difference was found for the mean number of mixed periods between session 1 [41\% (SD 7\%)] and session 4 [35 (SD 9)\%]. However, the variation in the exerted force during the contractions within one 100second recording period was higher $(\mathrm{P}<0.001)$ during the repetitive task than during the force reference task. 
Table 1. Perceived physical and mental exertion. The gray area indicates the periods with a repetitive task.

\begin{tabular}{|c|c|c|c|c|c|c|c|c|c|c|c|c|c|c|}
\hline \multirow{2}{*}{$\begin{array}{l}\text { Type of perceived } \\
\text { exertion a }\end{array}$} & \multicolumn{2}{|c|}{ Before task } & \multicolumn{2}{|c|}{ Session 1} & \multicolumn{2}{|c|}{ Session 2} & \multicolumn{2}{|c|}{ Session 3} & \multicolumn{2}{|c|}{ Session 4} & \multicolumn{2}{|c|}{ Recovery } & \multicolumn{2}{|c|}{$\mathrm{P}$-value } \\
\hline & Mean & SD & Mean & SD & Mean & SD & Mean & SD & Mean & SD & Mean & SD & Time $^{b}$ & Contractions ${ }^{c}$ \\
\hline \multicolumn{15}{|l|}{ Physical } \\
\hline \multicolumn{15}{|l|}{ Hand } \\
\hline During contractions & . & . & 3.2 & 1.5 & 3.6 & 1.4 & 4.6 & 1.1 & 5.2 & 1.0 & . & . & $<0.001$ & 0.001 \\
\hline Between contractions & 0.0 & 0.0 & 1.7 & 1.1 & 2.0 & 1.2 & 3.2 & 1.1 & 3.7 & 1.1 & 0.7 & 0.9 & . & . \\
\hline \multicolumn{15}{|l|}{ Forearm } \\
\hline During contractions & . & . & 2.7 & 1.5 & 2.9 & 1.7 & 3.6 & 1.1 & 4.1 & 1.2 & . & . & $<0.001$ & 0.003 \\
\hline Between contractions & 0.2 & 0.4 & 1.2 & 1.0 & 1.4 & 1.3 & 2.2 & 1.0 & 2.7 & 1.2 & 0.3 & 0.5 & . & . \\
\hline \multicolumn{15}{|l|}{ Shoulder } \\
\hline During contractions & . & . & 2.1 & 1.3 & 2.5 & 1.3 & 2.8 & 1.3 & 3.2 & 1.8 & . & . & $<0.001$ & 0.028 \\
\hline Between contractions & 0.2 & 0.4 & 1.0 & 0.7 & 1.5 & 1.1 & 1.9 & 1.1 & 2.0 & 1.5 & 0.4 & 0.9 & $\cdot$ & $\cdot$ \\
\hline \multicolumn{15}{|l|}{ Mental } \\
\hline Positive feelings & 6.8 & 2.2 & 7.3 & 1.4 & 7.1 & 1.4 & 6.7 & 1.9 & 6.5 & 1.9 & 6.7 & 2.1 & 0.13 & . \\
\hline Negative feelings & 1.1 & 1.1 & 2.7 & 1.6 & 3.1 & 1.6 & 3.3 & 1.9 & 2.9 & 1.3 & 1.0 & 1.0 & 0.50 & . \\
\hline Boring task & 2.2 & 1.6 & 3.6 & 2.1 & 4.8 & 2.2 & 5.5 & 2.7 & 5.1 & 2.5 & 3.5 & 2.2 & 0.10 & . \\
\hline Demanding task & 2.3 & 1.1 & 4.8 & 2.3 & 4.8 & 1.9 & 4.1 & 1.7 & 3.5 & 1.5 & 2.0 & 1.8 & 0.03 & . \\
\hline Funny task & 1.3 & 0.8 & 1.3 & 1.1 & 0.7 & 0.9 & 0.9 & 1.2 & 0.9 & 1.1 & 1.1 & 0.8 & 0.14 & . \\
\hline
\end{tabular}

a The scales were 0 to 9 for physical exertion (Borg scale) and 0 to 10 for mental exertion.

${ }^{b}$ Differences between times during the repetitive task (time).

c Differences during and between contractions.

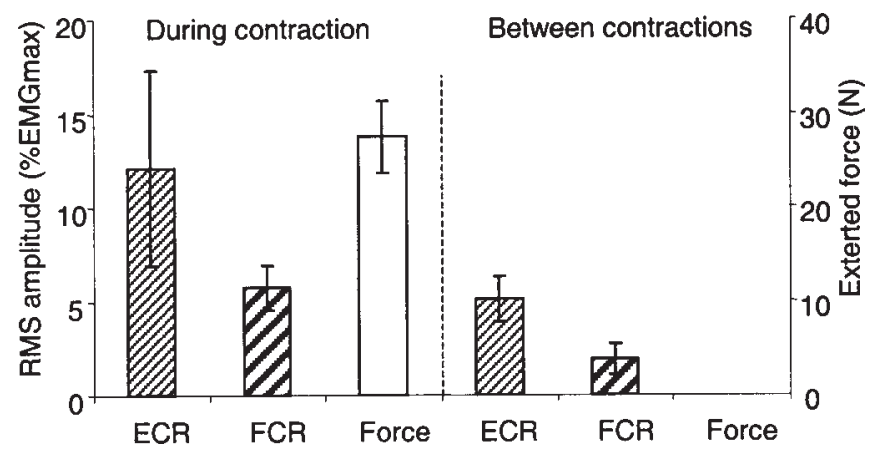

Figure 4. RMS (root mean square) amplitude and force during and between contractions. The results are the means and 95\% confidence intervals for six subjects for the extensor carpi radialis (ECR) and flexor carpi radialis (FCR) muscles (y-axis to the left) and force ( $y$-axis to the right). ( $E M G=$ electromyography)

\section{Electromyography}

The maximum RMS amplitudes measured during the handgrip MVC were 330 (SD 18) $\mu \mathrm{V}$ for the extensor carpi radialis muscle and 451 (SD 90) $\mu \mathrm{V}$ for the flexor carpi radialis muscle. No progressive changes were observed for the RMS amplitudes and the mean power frequency during the repetitive task. The mean RMS amplitudes, which were higher during contractions than between contractions for the extensor carpi radialis and flexor carpi radialis muscles, are presented in figure 4 . The mean power frequency during contractions was 113 (SD 12) $\mathrm{Hz}$ for the extensor carpi radialis muscle and 104 (SD 36) $\mathrm{Hz}$ for the flexor carpi radialis muscle in the beginning of session 1. The RMS amplitudes were higher $(\mathrm{P}=0.06$ and $\mathrm{P}=0.03$ for the extensor carpi radialis and flexor carpi radialis muscles, respectively) during the mental reference task [extensor carpi radialis muscle: 19.9 (SD 10.8)\% $\mathrm{EMG}_{\max }$; flexor carpi radialis muscle: 5.5 (SD 1.7)\% $\mathrm{EMG}_{\max }$ ] than during the repeti- tive task [the overall mean RMS amplitudes (during and between contractions) during the repetitive task being $7.0(\mathrm{SD} 1.3) \% \mathrm{EMG}_{\max }$ for the extensor carpi radialis msucle and $3.6(\mathrm{SD} 0.8) \% \mathrm{EMG}_{\max }$ for flexor carpi radialis muscle].

\section{Biomarkers}

A progressive increase was found for the norepinephrine concentrations during the repetitive task on the experimental day [+17 (95\% CI 7-29)\%/hour, $\mathrm{P}=0.002$; reference day included: $\mathrm{P}=0.06]$. There were no progressive increases for any of the other biomarkers measured in urine (figure 5) or blood. The epinephrine and norepinephrine concentrations in urine were higher to be higher [epinephrine: $+155 \%$ (95\% CI 69-283\%) $\mathrm{P}=0.002$; norepinephrine: $+77 \%$ (95\% CI 42-120\%) $\mathrm{P}<0.001]$ during the repetitive task than during the reference day (table 2), whereas there was a tendency for 

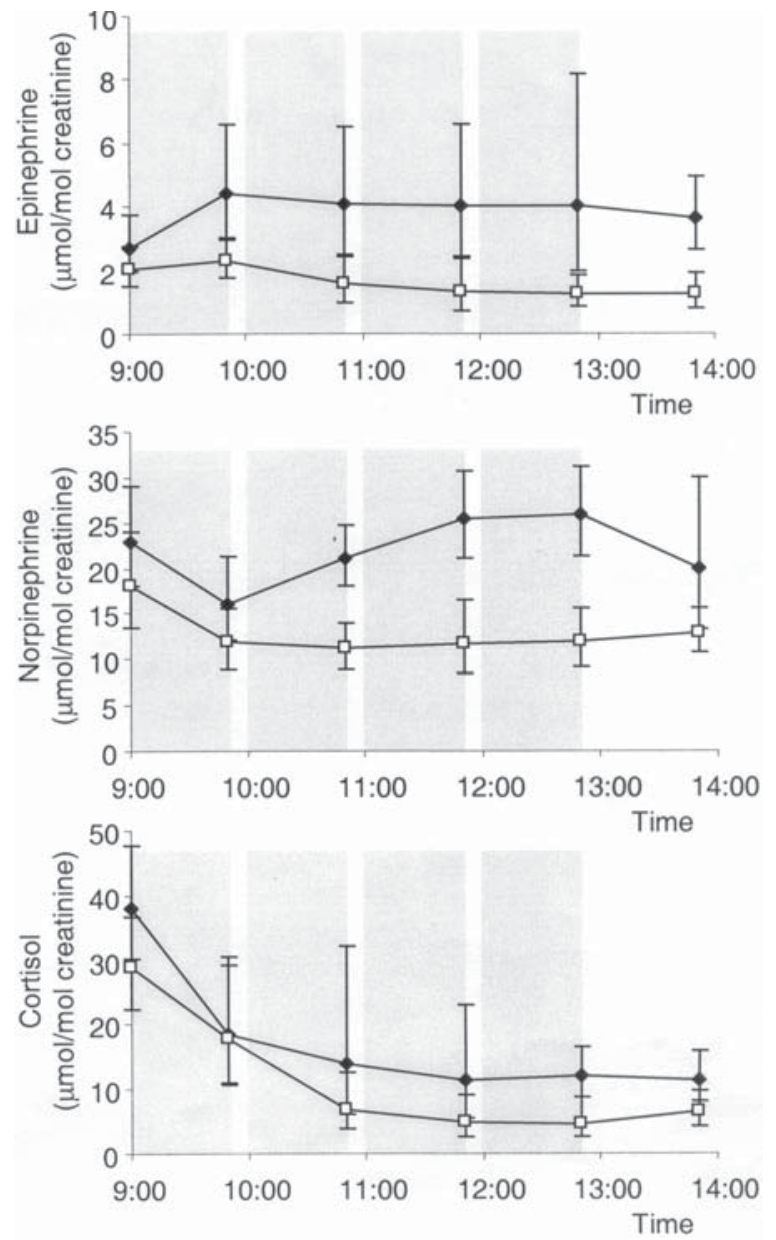

$\multimap-$ Experimental day $-\square-$ Reference day

Figure 5. Concentrations of urinary epinephrine, norepinephrine, and cortisol on the experimental and reference days. The results are the mean and $95 \%$ confidence intervals for nine subjects. The gray areas indicate the periods with a repetitive task.

cortisol to be higher $[+72 \%$ (95\% CI $1-191 \%)$, $\mathrm{P}=0.074]$. There were no differences between the morning concentrations of the biomarkers on the experimental day and the reference day, except for cortisol. There were no differences between the experimental day and the reference day for the concentrations of biomarkers in blood (table 2). A diurnal rhythm was observed on the reference day for concentrations of epinephrine $(\mathrm{P}=0.04)$ and cortisol $(\mathrm{P}=0.002)$ in urine (figure 5) and the concentrations of ACTH $(\mathrm{P}=0.08)$ and prolactin $(\mathrm{P}<0.001)$ in blood (table 2$)$.

\section{Discussion}

In our study we investigated potential accumulative effects on physiological responses during 4 hours of repetitive work under standardized conditions. There was a progressive increase in the concentrations of
Table 2. Concentrations of biomarkers in urine $(\mathrm{N}=9)$ and blood $(\mathrm{N}=10)$ on the experimental day and the reference day. The gray areas indicate periods with a repetitive task. $(95 \% \mathrm{Cl}=95 \%$ confidence interval, $\mathrm{ACTH}=$ adrenocorticotrophic hormone, DHEA-S = dehydroepiandrosterone sulfate, $\lg \mathrm{A}=$ immunoglobulin $\mathrm{A}$ )

\begin{tabular}{|c|c|c|c|c|c|c|}
\hline \multirow[t]{2}{*}{ Biomarker } & \multicolumn{2}{|c|}{$\begin{array}{l}\text { Experimental day } \\
\text { (repetitive task) }\end{array}$} & \multicolumn{2}{|c|}{$\begin{array}{l}\text { Reference } \\
\text { day }\end{array}$} & \multicolumn{2}{|c|}{ P-value } \\
\hline & Mean & $95 \% \mathrm{Cl}$ & Mean & $95 \% \mathrm{Cl}$ & Day a & $T \times D^{b}$ \\
\hline \multicolumn{7}{|c|}{ Urinary epinephrine ( $\mu \mathrm{mol} / \mathrm{mol}$ creatinine) } \\
\hline Before repetitive task & 2.7 & $1.0-7.1$ & 2.1 & $0.8-5.1$ & 0.09 & . \\
\hline During repetitive task & 4.1 & $1.1-15.0$ & 1.6 & $0.5-4.5$ & 0.002 & 0.74 \\
\hline After recovery & 3.6 & $1.4-9.1$ & 1.2 & $0.3-4.6$ & 0.01 & $\cdot$ \\
\hline \multicolumn{7}{|c|}{ Urinary norepinephrine ( $\mu \mathrm{mol} / \mathrm{mol}$ creatinine) } \\
\hline Before repetitive task & 22.8 & $11.1-46.8$ & 18.0 & $7.6-42.6$ & 0.16 & . \\
\hline During repetitive task & 21.7 & $14.2-33.2$ & 11.7 & $6.0-22.6$ & 0.001 & 0.002 \\
\hline After recovery & 19.9 & $5.9-67.8$ & 12.9 & $7.4-22.4$ & 0.06 & $\cdot$ \\
\hline \multicolumn{7}{|c|}{ Urinary cortisol ( $\mu \mathrm{mol} / \mathrm{mol}$ creatinine) } \\
\hline Before repetitive task & 37.9 & $18.9-75.9$ & 28.8 & $13.8-60.2$ & 0.03 & $\cdot$ \\
\hline During repetitive task & 13.6 & $2.9-64.5$ & 7.2 & $1.7-31.2$ & 0.07 & 0.23 \\
\hline After recovery & 11.4 & $4.3-30.3$ & 6.4 & $1.9-21.5$ & 0.003 & . \\
\hline \multicolumn{7}{|l|}{ Blood ACTH (pmol/l) } \\
\hline Before repetitive task & 3.7 & $1.3-10.2$ & 4.3 & $1.6-11.4$ & 0.20 & . \\
\hline During repetitive task & 3.1 & $1.4-6.9$ & 3.3 & $1.7-6.1$ & 0.77 & 0.02 \\
\hline After recovery & 2.7 & $1.7-4.2$ & 3.2 & $2.2-4.7$ & 0.08 & $\cdot$ \\
\hline \multicolumn{7}{|l|}{ Serum DHEA-S ( $\mu \mathrm{mol} / \mathrm{l})$} \\
\hline Before repetitive task & 7.7 & $3.7-16.0$ & 7.9 & $4.6-13.7$ & 0.67 & . \\
\hline During repetitive task & 7.7 & $4.7-12.7$ & 7.5 & $4.4-12.7$ & 0.68 & 0.21 \\
\hline After recovery & 7.9 & $3.2-19.3$ & 7.3 & $4.1-13.0$ & 0.27 & $\cdot$ \\
\hline \multicolumn{7}{|l|}{ Serum $\lg A(g / l)$} \\
\hline Before repetitive task & 2.1 & $0.8-5.1$ & 2.1 & $0.9-5.2$ & 0.45 & $\cdot$ \\
\hline During repetitive task & 2.1 & $0.8-5.3$ & 2.1 & $0.9-5.1$ & 0.56 & 0.14 \\
\hline After recovery & 2.1 & $0.8-5.5$ & 2.0 & $0.8-5.1$ & 0.21 & $\cdot$ \\
\hline \multicolumn{7}{|l|}{ Serum prolactin (mIU/l) } \\
\hline Before repetitive task & 270 & $123-592$ & 294 & $127-680$ & 0.65 & . \\
\hline During repetitive task & 118 & $39-360$ & 133 & $58-307$ & 0.61 & 0.02 \\
\hline After recovery & 134 & $46-390$ & 133 & $28-620$ & 0.96 & $\cdot$ \\
\hline \multicolumn{7}{|c|}{ Serum free-testosterone $(\mathrm{pmol} / \mathrm{l})$} \\
\hline Before repetitive task & 10.2 & $4.6-22.5$ & 10.3 & $4.8-22.0$ & 0.89 & $\dot{0}$ \\
\hline During repetitive task & 10.8 & $4.9-24.1$ & 9.2 & $3.8-22.5$ & 0.12 & 0.12 \\
\hline After recovery & 9.8 & $4.3-22.4$ & 9.0 & $3.5-23.3$ & 0.57 & $\cdot$ \\
\hline
\end{tabular}

aifferences between the experimental and reference days for data collected before, during, and after the repetitive task.

${ }^{b}$ Differences between times during the repetitive task on the experimental day.

urinary norepinephrine during the repetitive task. In contrast, no progressive changes were observed for epinephrine and cortisol in urine and ACTH, DHEA-S, immunoglobulin $\mathrm{A}$, prolactin or free testosterone in plasma or serum, except for those explainable by diurnal variation. Furthermore, there were no indications of accumulative EMG signs of fatigue in terms of increased RMS amplitudes or decreased mean power frequency or changes in cardiovascular responses during the repetitive task. When taken together, there were no indications of accumulative physiological effects (except for those on noradrenaline) during the 4-hour low-level repetitive task that simulated real life work by combining mental and physical demands. Thus exposure of 12 hours appeared to be adequate for determining the physiological responses, except for norepinephrine, to 
the low-level repetitive work performed for 4 hours in our study. However, longer exposure times and repeated sampling may be preferred due to increased statistical power.

In our study, the combination of low-level intermittent handgrip and attention demands resulted in considerable increases in urinary concentrations of epinephrine, norepinephrine, and cortisol when compared with the corresponding concentrations on the reference day. Two previous studies have shown that plasma concentrations of norepinephrine, but not epinephrine, increase in response to short-term dynamic handgrip at $25 \% \operatorname{MVC}(4,5)$. Due to considerable differences in physical demands and duration, comparisons with these studies must be made with caution. It could, however, indicate that the increase in urinary concentrations of, particularly, epinephrine observed in our study should be explained by other factors (eg, mental demands in addition to the physical task). Indeed low-level repetitive work with attention demands has been shown to cause increased urinary concentrations of epinephrine, norepinephrine, and cortisol $(9,10)$ in laboratory studies.

In contrast to the physiological measures there was an accumulative effect for the repetitive task, as reflected by a progressive increase in perceived physical exertion (table 1). A progressive decrease in the rating of how demanding the task was indicated that some degree of learning took place (table 1). However, the time spent at solving a mentally demanding task decreased during the repetitive task, whereas there were no differences in the observed number of errors. The increased speed indicates that the participants kept trying to do their best, despite some degree of learning.

Previously observed attention-related muscle activity (14) was also shown for forearm muscles in our study. This finding indicates that the effect of mental demands on muscle activity is a more general effect on all the active muscles, as observed in a recent study (15). Muscle activity was found in the periods between the contractions of the repetitive task although no external force was exerted and the forearm was fully supported. Furthermore, during the mental reference task, a high level of muscular activity was found, especially in the forearm extensor muscles, although the forces applied to the keyboard are expected to be less than $1 \mathrm{~N}$ (18). Hence, our study revealed no straightforward relationship between muscle activity and external force during combined attention-demanding low-level physical work. Therefore the study supported the hypothesis that the biomechanical need to stabilize body parts and factors that are not directly linked to biomechanical needs (eg, mental load, emotional load, and trait characteristics of the participant) also contribute to muscle activity (14). Thus assessments of workloads based primarily on external force measurements during a low-level repetitive task including demands for attention may lead to the wrong conclusions. Therefore, external force is an important, but not sufficient indicator of muscular load during low-level repetitive work.

The force exerted during contractions varied more when the participants performed the repetitive task than it did in the reference handgrip task, in which no attention-demanding computer task was performed. Thus our study indicated that work including high demands for attention may be performed at the expense of the precision of the exerted force. This phenomenon may have practical implications in that worktasks with high attention demands may lead to lower force precision.

In conclusion, concentrations of the urinary biomarkers were increased during the repetitive task. However, only the concentrations of norepinephrine showed a progressive increase during the 4 hours. In contrast, the perceived exertion in the hand, forearm, and shoulder regions increased progressively. Forearm muscle activity above the resting level was found in the periods in which no force was exerted and therefore indicated attention-related muscle activity. Finally, repetitive work including high demands for attention may be performed at the expense of the precision of the exerted force.

\section{Acknowledgments}

We would like to thank Niels Juel Christensen for his fruitful discussions and the ACTH analyses. Anne Abildtrup, Pia Birthe Jeppesen, Dorrit Meincke, and Ulla Tegner provided skilled technical assistance in the analyses of the biological material. Dorte Ekner provided technical assistance with the analyses of the EMG data.

The present study was supported by The Danish Research Academy (44-4425), The Danish Working Environment Fund (1994-08 S), and The Danish Heart Foundation.

\section{References}

1. Schwarz L, Kindermann W. Beta-endorfin, catecholamines, and cortisol during exhaustive endurance exercise. Int $\mathbf{J}$ Sports Med 1989;10:324-8.

2. Bonen A, Keizer HA. Pituitary, ovarian and adrenal hormone responses to marathon running. Int J Sports Med 1987;8:1617.

3. Scavo D, Barletta C, Vagiri D, Letizia C. Adrenocorticotropic hormone, beta-endorphin, cortisol, growth hormone and prolactin circulating levels in nineteen athletes before and after half-marathon and marathon. J Sports Med Phys Fitness 
1991;31:401-6.

4. Lewis SF, Taylor WF, Bastian BC, Graham RM, Pettinger WA, Blomqvist CG. Haemodynamic responses to static and dynamic handgrip before and after autonomic blockade. Clin Sci 1983;64:593-9.

5. Sinoway L, Shenberger J, Leaman G, Zelis R, Gray K, Baily $\mathrm{R}$, et al. Forearm training attenuates sympathetic responses to prolonged rhythmic forearm exercise. J Appl Physiol 1996; 81:1778-84.

6. Rissén D, Melin B, Sandsjö L, Dohns I, Lundberg U. Surface EMG and psychophysiological stress reactions in women during repetitive work. Eur J Appl Physiol 2000;83:215-22.

7. Melin B, Lundberg U, Söderlund J, Granqvist M. Psychological and physiological stress reactions of male and female assembly workers: a comparison between two different forms of work organization. J Organ Behav 1999;20:47-61.

8. Lundberg U, Melin B, Evans GW, Holmberg L. Physiological deactivation after two contrasting tasks at a video display terminal: learning vs repetitive data entry. Ergonomics 1993;36:601-11.

9. Lundberg U, Frankenhaeuser M. Pituitary-adrenal and sympathetic-adrenal correlates of distress and effort. J Psychosom Res 1980;24:125-30.

10. Frankenhaeuser M, Nordheden B, Myrsten A, Post B. Psychophysiological reactions to understimulation and overstimulation. Acta Psychol 1971;35:298-308.

11. Garde AH, Laursen B, Jørgensen AH, Jensen BR. Effects of mental and physical demands on heart rate variability during computer work. Eur J Appl Physiol 2002;87:456-61.

12. Weber A, Fussler C, O'Hanlon JF, Gierer R, Grandjean E. Psychophysiological effects of repetitive tasks. Ergonomics 1980;23(11):1033-46.

13. O'Hanlon JF, Beatty J. Catecholamine correlates of radar monitoring performance. Biol Psychol 1976;4:293-304.

14. Wærsted M. Human muscle activity related to non-biomechanical factors in the workplace. Eur J Appl Physiol 2000;83:151-8.

15. Laursen B, Jensen BR, Garde AH, Jørgensen AH. Effect of mental and physical demands on muscular activity during the use of a computer mouse and a keyboard. Scand J Work Environ Health 2002;28(4):215-21.

16. van Breukelen GJP, Roskam EECI, Eling PATM, Jansen RWTL, Souren DAPB, Ickenroth JGM. A model and diagnostic measures for response time series on tests of concen- tration: historical background, conceptual framework and some applications. Brain Cogn 1995;27:147-70.

17. Wærsted M, Bjørklund RA, Westgaard RH. The effect of motivation on shoulder-muscle tension in attention-demanding task. Ergonomics 1994;37:363-76.

18. Gerard MJ, Armstrong TJ, Franzblau A, Martin BJ, Rempel DM. The effects of keyswitch stiffness on typing force, finger electromyography, and subjective discomfort. Am Ind Hyg Assoc J 1999;60:762-9.

19. Borg GAV. Psychophysical bases of perceived exertion. Med Sci Sports Exerc 1982;14:377-81.

20. Kwatny E, Thomas DH, Kwatny HG. An application of signal processing techniques to the study of myoelectric signals. IEEE Trans Biomed Eng 1970;17:303-12.

21. Hansen ÅM, Kristiansen J, Nielsen JL, Byrialsen K, Christensen JM. Validation of a high performance liquid chromatography analysis for the determination of noradrenaline and adrenaline in human urine with an on-line sample purification. Talanta 1999;50:367-79.

22. Bartels H, Bohmer M. Eine mikrometode zur kreatininbestimmung. Clin Chim Acta 1971;32:81-5.

23. Hansen AM, Garde AH, Christensen JM, Eller NH, Netterstrøm B. Reference intervals and variation for urinary epinephrine, norepinephrine and cortisol in healthy men and women in Denmark. Clin Chem Lab Med 2001;39:842-9.

24. Hansen ÅM, Garde AH, Christensen JM, Eller N, Netterstrøm B. Validation of a radio-immunoassay and establishment of a reference interval for salivary cortisol in healthy subjects in Denmark. Scand J Clin Lab Invest 2003;63:1-9.

25. Garde AH, Hansen AM. Evaluation of enzyme-linked immuno-sorbant assay (ELISA) for measurement of dehydroepiandrosterone-sulphate (DHEA-S) in serum of healthy women [abstract]. In: Aitio A, Engström K, Kiilunen M, Liesivuori J, Pyy L, Riihimäki V, editors. International Symposium on Biological Monitoring in Occupational and Environmental Health, Espoo, Finland. Helsinki: Finnish Institute of Occupational Health; 1996. p 233-4.

26. Garde AH, Hansen AM, Skovgaard LT, Christensen JM. Seasonal and biological variation of blood concentrations of total cholesterol, dehydroepiandrosterone sulfate, hemoglobin $\mathrm{A}(1 \mathrm{c}), \operatorname{Ig} \mathrm{A}$, prolactin, and free testosterone in healthy women. Clin Chem 2000;46:551-9.

Received for publication: 13 December 2002 